\title{
THE UNIFICATION OF RADIO-LOUD AGN
}

\author{
C. M. URRY
}

Space Telescope Science Institute

3700 San Martin Drive, Baltimore, Maryland, 21218

AND

\section{PAOLO PADOVANI}

Dipartimento di Fisica, II Università di Roma "Tor Vergata" Via della Ricerca Scientifica 1, I-00133 Roma

\section{Summary}

In a recent review paper we summarized the current status of unification of radio-loud AGN (Urry \& Padovani 1995 PASP 107, 803), connecting high-luminosity (FR II) radio galaxies with quasars, and low-luminosity (FR I) radio galaxies with BL Lac objects. Unified schemes are motivated by the knowledge that AGN appearance depends strongly on orientation (Fig. 1): optical/UV light from the centers of many AGN is obscured by circumnuclear matter, and in radio-loud AGN, bipolar relativistic jets beam light along the jet axes. Understanding these radiation anisotropies allows us to unify apparently distinct classes of AGN that differ primarily because of orientation.

Our review described the classification and general properties of AGN and summarized the evidence for anisotropic emission caused by circumnuclear obscuration and relativistic beaming. We outlined the evidence, both observed isotropic properties and statistical arguments, for connecting FR IIs with quasars and FR Is with BL Lacs. The population statistics (with beaming) are in accordance with available data and suggest $\gamma \sim 5$ for low-luminosity AGN and $\gamma \sim 10$ for high-luminosity AGN. The distinctions between X-ray-selected and radio-selected BL Lac objects, and between BL Lacs and flat-spectrum variable quasars, still not understood, provide clues to the underlying physics of blazars. Our review discussed several possible problems and complications, and concluded with a list of the ten questions we believe are the most pressing in this field. 


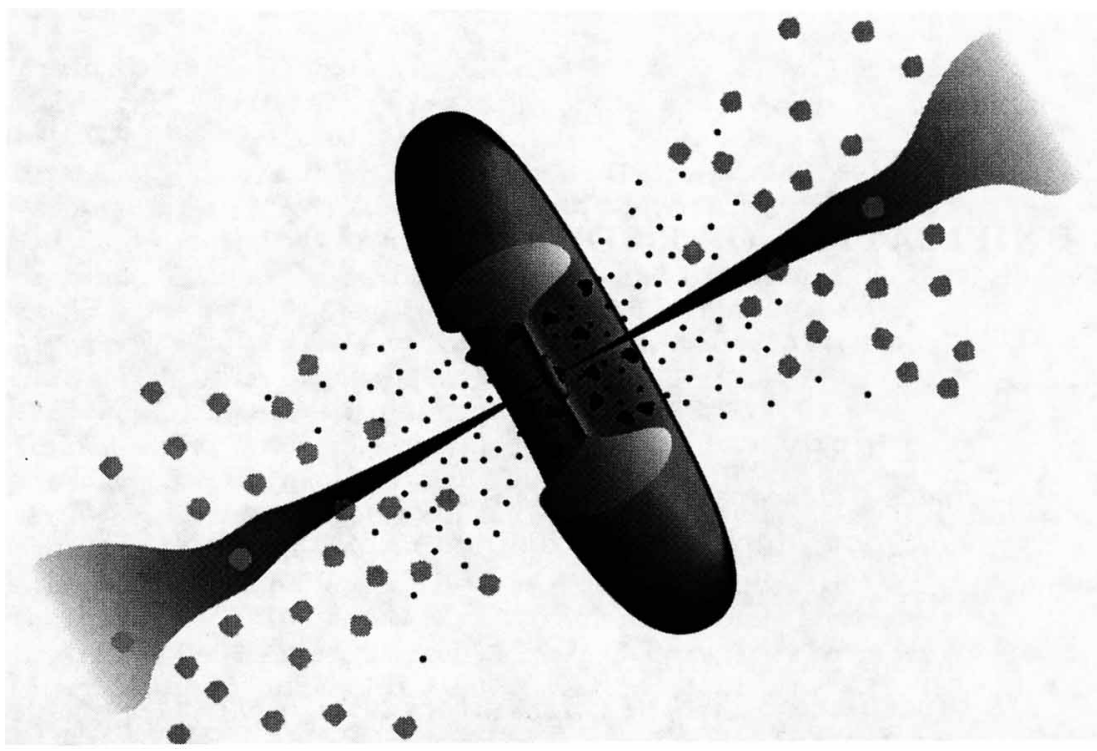

Figure 1. Current paradigm for radio-loud AGN: central black hole (scales for $10^{8} \mathcal{M} \odot$ black hole, $\left.R \sim 3 \times 10^{13} \mathrm{~cm}\right)$, surrounded by accretion disk $\left(r \sim 1-30 \times 10^{14} \mathrm{~cm}\right)$, broad-line clouds $\left(\sim 2-20 \times 10^{16} \mathrm{~cm}\right.$ from $\left.\mathrm{BH}\right)$, thick dusty torus obscuring transverse lines of sight (inner radius $\left.\sim 10^{17} \mathrm{~cm}\right)$, and narrow line clouds $\left(10^{18}-10^{20} \mathrm{~cm}\right)$. Hot electrons scatter continuum and BLR photons into line of sight and may produce hard X-ray continuum. Radio jets (shown as diffuse FR I-type) are relativistic near the $\mathrm{BH}\left(10^{17}\right)$, produce relativistically beamed optical through $\gamma$-ray emission in the inner regions, and can extend to several times $10^{24} \mathrm{~cm}$;

\section{THE TEN MOST IMPORTANT QUESTIONS}

1. Are BL Lacs or obscured quasars in all radio galaxies?

2. What is the relation between BL Lacs and FSRQ?

3. Are superluminal velocities, core-to-extended flux ratios, and jet/counter-jet ratios commensurate with beaming?

4. Is the bulk Lorentz factor higher in high-luminosity radio sources (quasars, FR IIs) than in low-luminosity radio sources (BL Lacs, FR Is)?

5. Do FR Is have broad emission line regions?

6. What is the relation between FR Is and FR IIs?

7. How do jets form and propagate?

8. What is the physical cause of the radio loud - radio quiet distinction?

9. Where are the narrow-line (Type 2) radio-quiet quasars?

10. What are the fundamental parameters governing the central engine, and is it powered by a black hole? 